\title{
Development of Analytical Model for Dynamic Bending Force during Single Pass 3-roller Cone Frustum Bending Technique
}

\author{
Mahesh Chudasama $^{1, *}$, Harit Raval $^{2}$ \\ ${ }^{1}$ Ghandhy Government Engineering College, Surat (Gujarat) India \\ ${ }^{2}$ National Institute of Technology, Surat (Gujarat) India \\ *Corresponding Author: mkcgecs@gmail.com
}

Copyright $(2014$ Horizon Research Publishing All rights reserved.

\begin{abstract}
3-roller conical bending process is one which is used for producing conical sections or cone frustums from metal plates for various industrial applications. The plates to be bent are cut for the required size and shape and then rolled between three cylindrical rollers. The plate is kept between one top roller and two bottom rollers. Than the top roller is lowered down and the plate gets bend. The rollers are rotated to get the roll bending of the plate. Final dimension of the cone frustum are achieved through setting appropriate machine setting parameters. An attempt is made in this paper to develop the analytical model for the prediction of the bending force during the stage of dynamic roll bending. During the roll bending of the plate there will be shear stresses along with the normal stresses. The analytical model has been developed considering these shear stresses. The model consists of various parameters like material parameters and geometrical parameters. Based on the analytical models derived, effects of some of the material parameters on dynamic bending force have been studied. In the development process complex mechanics involved in this process has been simplified so it will give insight of the process and will be helpful to the researchers and designers working in the area of metal forming, especially in roll bending process.
\end{abstract}

Keywords Cone-Frustum, Dynamic-Roll-Bending, Force-Prediction, Analytical Model

\section{Introduction}

In modern world, every human being depends on technology for one or the other reason. It can be day to day life requirements, luxury or for entertainment. Manufacturing is one of the technologies to satisfy such human requirements. Though metal forming is an age old manufacturing technology, it is still in demand due to its versatility in satisfying the human needs. If there can be small reduction in cost or the increase in the effectiveness of any of the metal forming processes, it will positively affect the metal forming industry and hence the economy in the larger context.

Metal forming process in which straight line of the metal is transformed into a curved length is called bending. Roll bending or Roller forming is a continuous bending operation in which a long strip of metal is passed through consecutive sets of rollers or a roller stand, each performing only an incremental part of the bend, until the desired cross sectional profile is obtained. It is observed that the roller bending process usually produces higher dimensional accuracy of the finished products [1].

Conical shells and sections are also widely used in manufacturing of air duct transitions, chimneys, fan inlets, towers for wind mills, hull of ships, structural components of on shore oil and gas platforms etc. These conical shells and sections are widely used in process industries. 3-roller conical bending machines are used for production of such conical shells and sections from the metal plates.

Conical bending using 3-roller bending machine can be achieved by either setting the top rollers inclined in vertical plane or setting the bottom rollers inclined in horizontal plane. Conical bending can also achieved by inclining the top roller and bottom rollers simultaneously. Bottom roller inclination significantly affects the bending moment as compared to top roller inclination during 3-roller conical bending [2] and hence, only bottom roller inclination in the horizontal plane is considered in the present analysis. In 3 -roller conical bending process, the bottom rollers are inclined as shown in figure 1 and the blank is kept between the top roller and the bottom rollers such that one of the generators of the cone frustum remains parallel to the bending axis. In case of 3-roller bending machine, the bending line lies parallel to the top roller axis. The top roller is than lowered down to get the static bending of the plate. At this stage the plate gets bend locally at the bending line, kept 
at one of the generator of the cone frustum. In the next stage the bottom rollers are driven with the help of electric motors and the plate gets dynamically bent throughout its length. The bottom rollers are rotated in the reverse direction once the complete length of the plate is bent and then the plate is unloaded.

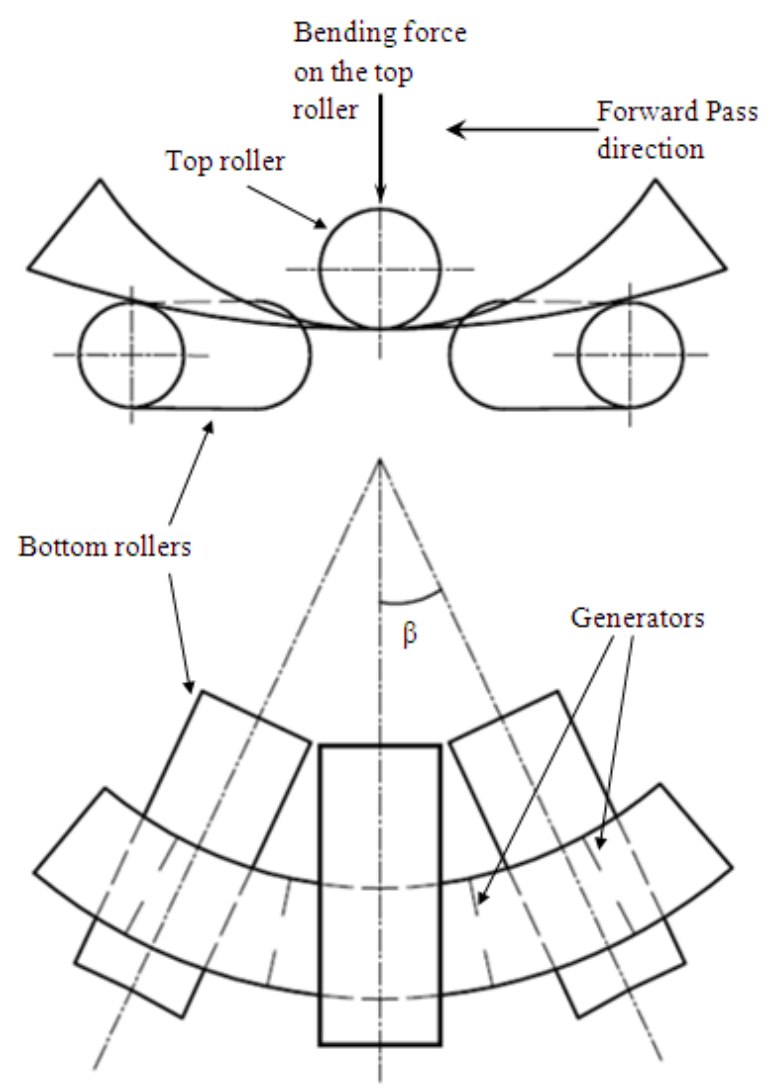

Figure 1. Schematic of 3-roller bending process

Research related to V-bending as well as roll bending for springback prediction has been reported extensively $[3,4,5,6,7]$. Hua et al. have extensively analyzed the 4-roll bending process considering various aspects like mechanics of the process, springback, internal and external moments, etc $[8,9,1]$. Raval reported the study of bendability of plate along with the simulation program to predict the desired top roller position to bend the plate at the required curvature on three-roller plate bending machine [10]. Gandhi et al have derived equations for Moment required for bending of blank with initial radius without applied tension, Springback for cone frustum bending, Top roller position for desired residual radius at reference and larger ends, Effective center distance between bottom rollers for desired residual radius at larger end [11,2]. Attempt for development of bending force prediction during static bending stage have been reported [12] but bending force prediction models for dynamic bending stage during 3-roller conical bending have not been reported as per the best of the knowledge of the authors. Hence an attempt is made in the present paper to develop such bending force prediction model for dynamic bending stage during 3-roller conical bending process.

\section{Analytical Model for Bending Force during Dynamic Roll Bending}

For development of force relation for dynamic bending stage, external and internal bending moments have been equated. Development of external bending moment for dynamic bending stage is discussed in next section.

\subsection{External Bending Moment during Dynamic Roll-bending Process}

After the completion of static bending of the plate when the bottom rollers are rotated in one direction using power drives, the roller will exert the forces over the plate to bend the plate in dynamic conditions. The forces exerted by the rollers will create the bending moment which will bend the plate. There will be normal reaction forces as well as frictional forces at the roller-plate interface. Schematic arrangement of the rollers and the plate with the help of cross section of the roller-plate during dynamic bending conditions is shown in figure 2 . The direction of the reaction forces over the rollers as well as the frictional forces is shown in figure 2 .
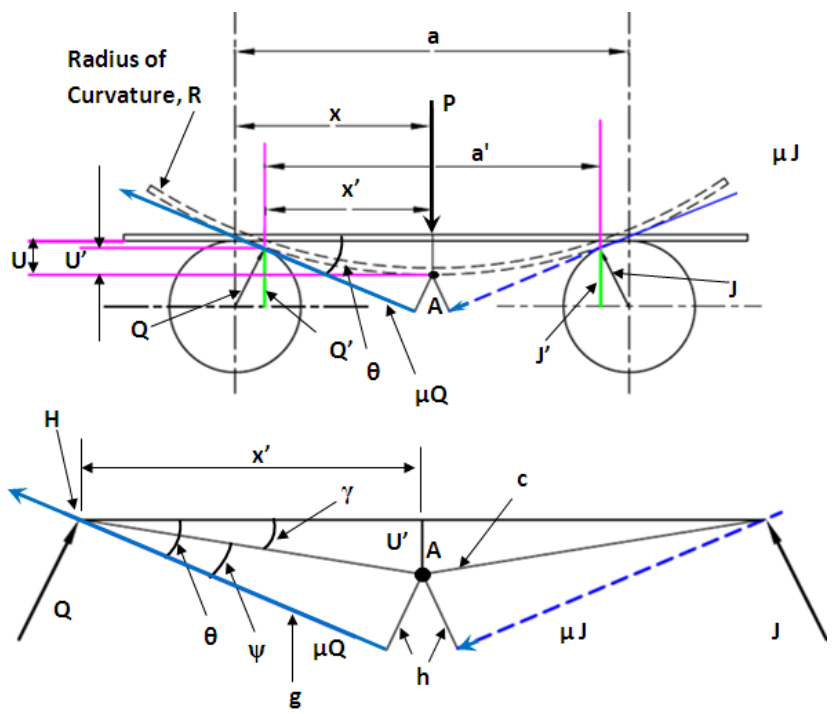

Figure 2. Schematic of blank and roller arrangement for dynamic roll-bending

Here bottom roller rotation is considered to be anti-clockwise in figure 2. Cross section of the roller set up at some radius of blank $\mathrm{R}$ is shown. Top roller is not shown in the figure but the vertical reaction at the top roller and plate interface, $\mathrm{P}$ is shown. Bottom roller inclination can be seen in figure 1. But it cannot be visualised in figure $\mathrm{s}$ as the front view is shown and the cross section of the roller and plate along the width of the blank at bending radius $\mathrm{R}$ is taken. For particular bottom roller inclination, bending radius at front end and rear end will have certain values. Thus bottom roller inclination will affect the value of $\mathrm{R}$ along the width of the blank. In figure 2 reaction at the top roller i.e. bending force $\mathrm{P}$ at the top roller is shown. Top roller is given the displacement $U$ in the first stage of the static bending. 
Normal reaction on the bottom roller on left side by the plate is Q. As in dynamic bending stage rollers are rotated (anti-clockwise in the present case) the frictional force will be assisting the rollers to move the plate. So frictional force on the left hand side roller $\mu \mathrm{Q}$ will be leftwards and tangent to the roller surface as shown in figure 2. Normal reaction and frictional force for bottom right roller is shown by $\mathrm{J}$ and $\mu \mathrm{J}$ respectively. Frictional force on the right hand side roller $\mu \mathrm{J}$ will be leftwards and tangent to the right hand side roller as shown in figure 2. Other geometrical parameters for dynamic bending are also shown in the figure 2 .

For roll bending of the blank as shown in figure 2, bending moment at point A (i.e. point at an intersection of the line of action of the concentrated load \& outer fibre of the blank) considering the normal reactions at the roller plate interface can be given by,[13]

$$
\begin{gathered}
M_{\text {ext }}=Q g \\
M_{\text {ext }}=P\left(1-\frac{x-r_{1} \sin \theta}{a-r_{1} \sin \theta}\right)(1 / 1+\mu \tan \theta)\left(x-\tan \theta\left(r_{1}-U\right)\right)
\end{gathered}
$$

Now, total bending moment required to bend the plate also consists of the bending due to frictional forces. During dynamic roll bending of the plate the radius of the curvature of the plate is not constant. In such conditions from figure 2 it can be said that perpendicular distance of frictional forces $\mu \mathrm{Q}$ and $\mu \mathrm{J}$ will be different. But for present study the curvature of the bend plate is assumed constant and hence perpendicular distance for both the frictional forces from the point A will be same. It is designated by $\mathrm{h}$ in figure 2 .

Now external bending moment due to friction at point $A$, from figure 2,

$$
\begin{aligned}
& M_{\text {friction }}=\mu * Q * h+\mu * J * h \\
& M_{\text {friction }}=\mu(Q+J) h \\
& M_{\text {friction }}=\frac{\mu}{\cos \theta} * P * h
\end{aligned}
$$

Proceeding further, total bending moment can be derived as,

$$
M_{\text {total }}=2 M_{\text {ext }}+M_{\text {friction }}
$$

Replacing equation 2 and 4 in equation $5 \&$ simplifying;

$$
M_{\text {total }}=P\left[\begin{array}{l}
\left\{\left(1-\frac{x^{\prime}}{a^{\prime}}\right)\left(\frac{x-\tan \theta\left(r_{1}-U\right)}{1+\mu \tan \theta}\right)\right\} \\
-\left\{\frac{\mu x^{\prime}}{\cos \theta}\left(\sin \theta-\frac{x^{\prime} \cos \theta}{U^{\prime}}\right)\right\}
\end{array}\right]
$$

Where, $\quad a^{\prime}=a-r_{1} \sin \theta ; U^{\prime}=U-r_{1}(1-\cos \theta) ; x^{\prime}=$ $x-r_{1} \sin \theta$

Equation 6 gives the total external bending moment exerted by the rollers over the plate.

After getting the equation for external bending moment it is required to get the internal bending moment equation for the dynamic bending process.

\subsection{Internal Bending Considering Shear Stresses Along with Normal Stresses}

There will be 3-dimensional stress pattern as can be seen in figure 3. Along with normal stresses there will be shear stresses acting over the plane. If we consider infinitesimal element of the plate and show the normal axis by $\mathrm{x}, \mathrm{y}$ and $\mathrm{z}$ as shown in figure 3. Because of such 3-dimensional stress pattern there will be shear stress components along with the normal force for the stress tensor.
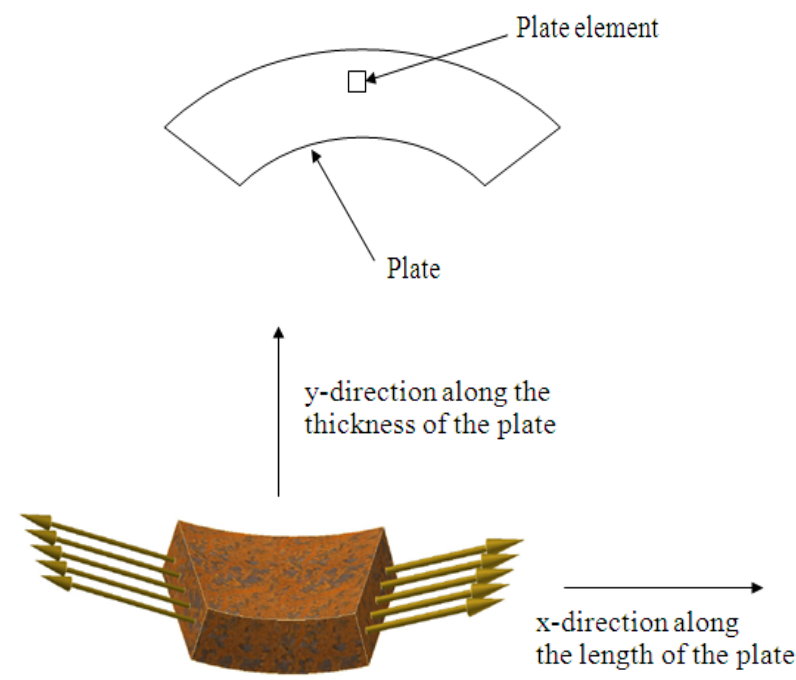

Figure 3. Plate element showing the 3-dimensional stress pattern

To get the internal bending moment equation, it is required to have the relation of effective stress and effective strain with normal stresses and normal strains respectively. For stress conditions as per theory proposed by Hill [14] and discussed by Hasek [15], effective stresses can be related with corresponding normal stresses by;

$$
\begin{aligned}
\bar{\sigma}=\left[\frac{3}{2} \frac{1}{F+G+H}\right. & \left\{F\left(\sigma_{y}-\sigma_{z}\right)^{2}+G\left(\sigma_{z}-\sigma_{x}\right)^{2}\right. \\
+ & H\left(\sigma_{x}-\sigma_{y}\right)^{2}+2 L \tau_{y x}^{2}+2 M \tau_{z x}^{2} \\
+ & \left.\left.2 N \tau_{y z}^{2}\right\}\right]^{\frac{1}{2}}
\end{aligned}
$$

For planar isotropy

$$
\begin{gathered}
\mathrm{F}=\mathrm{G}=\mathrm{H} \& \mathrm{~L}=\mathrm{M}=\mathrm{N}=3 \mathrm{~F}[16] \\
\bar{\sigma}=\left[\frac { 1 } { 2 } \left\{\left(\sigma_{y}-\sigma_{z}\right)^{2}+\left(\sigma_{z}-\sigma_{x}\right)^{2}+\left(\sigma_{x}-\sigma_{y}\right)^{2}+\right.\right. \\
6 \tau y x 2+\tau z x 2+\tau y z 212
\end{gathered}
$$

For 3-roller conical bending process there are no constraints in the width directions of the plate and hence $\sigma_{z}=0$ can be assumed. Also it is assumed that the thickness of the plate remains constant. So the strain element in thickness direction will also be very small and can be neglected. Hence the elements of the stress tensor, $\sigma_{z}=0$ $\& \tau_{x y}=0$, putting theses values in equation 7 ,

$$
\begin{gathered}
\bar{\sigma}=\left[\frac{1}{2}\left\{\left(\sigma_{y}\right)^{2}+\left(\sigma_{x}\right)^{2}+\left(\sigma_{x}-\sigma_{y}\right)^{2}+6\left(\tau_{z x}^{2}+\tau_{y z}^{2}\right)\right\}\right]^{\frac{1}{2}} \\
\bar{\sigma}=\left[\sigma_{x}{ }^{2}\left\{1+\zeta^{2}-\zeta\right\}+3\left(\tau_{z x}^{2}+\tau_{y z}^{2}\right)\right]^{\frac{1}{2}}
\end{gathered}
$$


Where, $\zeta=\sigma_{y} / \sigma_{x}$ (stress ratio) [17]

Now the relation for effective strain and normal strain can be obtained by considering the theory proposed by Hill [14] and discussed by Hasek [15]. The suggested relation of effective strain with corresponding normal strains is,

$$
\bar{\varepsilon}=\left[\frac{2}{3}(F+G+H)\right]^{\frac{1}{2}}\left[\left\{\frac{F\left(G \varepsilon_{z}-H \varepsilon_{x}\right)^{2}+G\left(F \varepsilon_{y}-H \varepsilon_{z}\right)^{2}+H\left(F \varepsilon_{x}-G \varepsilon_{y}\right)^{2}}{(F G+G H+H F)^{2}}\right\}+\right.
$$

Again for planar isotropy $\mathrm{F}=\mathrm{G}=\mathrm{H} \& \mathrm{~L}=\mathrm{M}=\mathrm{N}=3 \mathrm{~F}[18]$

$$
\bar{\varepsilon}=[2 F]^{\frac{1}{2}}\left[\left\{\frac{\left(\varepsilon_{z}-\varepsilon_{x}\right)^{2}+\left(\varepsilon_{y}-\varepsilon_{z}\right)^{2}+\left(\varepsilon_{x}-\varepsilon_{y}\right)^{2}}{9 F^{2}}\right\}+\left\{\frac{2 \gamma_{x y}^{2}}{3 F}+\frac{2 \gamma_{y z}^{2}}{3 F}+\frac{2 \gamma_{z x}^{2}}{3 F}\right\}\right]^{\frac{1}{2}}
$$

For Normal isotropy $\mathrm{F}=1[18]$

$$
\bar{\varepsilon}=[2]^{\frac{1}{2}}\left[\left\{\frac{\left(\varepsilon_{z}-\varepsilon_{x}\right)^{2}+\left(\varepsilon_{y}-\varepsilon_{z}\right)^{2}+\left(\varepsilon_{x}-\varepsilon_{y}\right)^{2}}{9}\right\}+\left\{\frac{2 \gamma_{x y}^{2}}{3}+\frac{2 \gamma_{y z}^{2}}{3}+\frac{2 \gamma_{z x}^{2}}{3}\right\}\right]^{\frac{1}{2}}
$$

For the case of conical bending, as the thickness is assumed to be constant, strain in y direction will be zero, hence $\varepsilon_{y}=0$ and $\gamma_{z x}=0$

$$
\bar{\varepsilon}=\left[\frac{2}{3}\right]^{\frac{1}{2}}\left[\left\{\frac{\left(\varepsilon_{z}\right)^{2}+\left(\varepsilon_{x}\right)^{2}-2 \varepsilon_{z} \varepsilon_{x}+\left(\varepsilon_{z}\right)^{2}+\left(\varepsilon_{x}\right)^{2}}{3}\right\}+\left\{2 \gamma_{x y}^{2}+2 \gamma_{y z}^{2}\right\}\right]^{\frac{1}{2}}
$$

Considering volume constancy, as $\varepsilon_{y}=0$ it can be derived as, $\varepsilon_{z}=-\varepsilon_{x}$

$$
\bar{\varepsilon}=\frac{2}{\sqrt{3}}\left[\left(\varepsilon_{x}\right)^{2}+\gamma_{x y}^{2}+\gamma_{y z}^{2}\right]^{\frac{1}{2}}
$$

The bending moment can be split into an elastic contribution and plastic contribution and can be calculated from [9],

$$
\begin{gathered}
M_{\text {total }}=M_{\text {elastic }}+M_{\text {plastic }} \\
=2 \int_{\text {elastic }} \sigma_{x} y d y+2 \int_{\text {plastic }} \sigma_{x} y d y
\end{gathered}
$$

For elastic portion of the internal bending moment,

$$
\begin{gathered}
\bar{\sigma}=E \bar{\varepsilon} \\
{\left[\sigma_{x}{ }^{2}\left\{1+\zeta^{2}-\zeta\right\}+3\left(\tau_{z y}^{2}+\tau_{z x}^{2}+\tau_{y z}^{2}\right)\right]^{\frac{1}{2}}} \\
=E \frac{2}{\sqrt{3}}\left[\left(\varepsilon_{x}\right)^{2}+\gamma_{x y}^{2}+\gamma_{y z}^{2}\right]^{\frac{1}{2}} \\
\sigma_{x}=\left[\frac{\left(E \frac{2}{\sqrt{3}}\right)^{2}}{\left\{1+\zeta^{2}-\zeta\right\}}\left[\left(\varepsilon_{x}\right)^{2}+\gamma_{x y}^{2}+\gamma_{y z}^{2}\right]-\frac{3\left(\tau_{z y}^{2}+\tau_{z x}^{2}+\tau_{y z}^{2}\right)}{\left\{1+\zeta^{2}-\zeta\right\}}\right]^{\frac{1}{2}}
\end{gathered}
$$

Where, $\zeta=\frac{\sigma_{y}}{\sigma_{x}}$ (constant) [17]

$$
\begin{aligned}
& \int_{0}^{y_{e p}} \sigma_{x} y d y=\int_{0}^{y_{e p}}\left[\frac{\left(E \frac{2}{\sqrt{3}}\right)^{2}}{\left\{1+\zeta^{2}-\zeta\right\}}\left[\left(\frac{y}{R}\right)^{2}+\gamma_{x y}^{2}+\gamma_{y z}^{2}\right]-\right. \\
& 3 \tau z y 2+\tau z x 2+\tau y z 21+\zeta 2-\zeta 12 y d y
\end{aligned}
$$

For development of the internal bending moment it is assumed that the material follows Power law material behavior and elastic-plastic bending conditions prevailing in the plastic region. So relation of effective stress and strain can be given as:

$$
\begin{gathered}
\bar{\sigma}=C \bar{\varepsilon}^{n} \dot{\varepsilon}^{\dot{m}} \\
{\left[\sigma_{x}{ }^{2}\left\{1+\zeta^{2}-\zeta\right\}+3\left(\tau_{z y}^{2}+\tau_{z x}^{2}+\tau_{y z}^{2}\right)\right]^{\frac{1}{2}}} \\
=C \frac{2}{\sqrt{3}}\left(\left[\left(\varepsilon_{x}\right)^{2}+\gamma_{x y}^{2}+\gamma_{y z}^{2}\right]^{\frac{1}{2}}\right)^{n} \dot{\varepsilon}^{\dot{m}}
\end{gathered}
$$

$\sigma_{x}=\left[\frac{\left(c \frac{2}{\sqrt{3}} \dot{\varepsilon}^{\dot{m}}\right)^{2}}{\left\{1+\zeta^{2}-\zeta\right\}}\left(\left[\left(\varepsilon_{x}\right)^{2}+\gamma_{x y}^{2}+\gamma_{y z}^{2}\right]^{\frac{1}{2}}\right)^{2 n}-\frac{3\left(\tau_{z y}^{2}+\tau_{z x}^{2}+\tau_{y z}^{2}\right)}{\left\{1+\zeta^{2}-\zeta\right\}}\right]^{\frac{1}{2}}$

$$
\begin{gathered}
\int_{y_{e p}}^{\frac{t}{2}} \sigma_{x} y d y=\int_{y_{e p}}^{\frac{t}{2}}\left[\frac{\left(c \frac{2}{\sqrt{3}} \dot{\xi} \dot{m}\right)^{2}}{\left\{1+\zeta^{2}-\zeta\right\}}\left(\left[\left(\frac{y}{R}\right)^{2}+\gamma_{x y}^{2}+\gamma_{y z}^{2}\right]^{\frac{1}{2}}\right)^{2 n}-\right. \\
3 \tau z y 2+\tau z x 2+\tau y z 21+\zeta 2-\zeta 12 y d y
\end{gathered}
$$

\subsection{Analytical Expression for Dynamic Bending Force Considering Shear Stresses Along With The Normal Stresses}

Dynamic bending force, $\mathrm{P}$ for the case considered here can be derived by external bending moment from equation 6 and internal bending moment from equation 20 as below:

$$
\begin{aligned}
& P\left[\left\{\left(1-\frac{x^{\prime}}{a^{\prime}}\right)\left(\frac{x-\tan \theta\left(r_{1}-U\right)}{1+\mu \tan \theta}\right)\right\}-\left\{\frac{\mu x^{\prime}}{\cos \theta}\left(\sin \theta-\frac{x^{\prime} \cos \theta}{U^{\prime}}\right)\right\}\right]= \\
& \int_{0}^{y_{e p}}\left[\frac{\left(E \frac{2}{\sqrt{3}}\right)^{2}}{\left\{1+\zeta^{2}-\zeta\right\}}\left[\left(\frac{y}{R}\right)^{2}+\gamma_{x y}^{2}+\gamma_{y z}^{2}\right]-\frac{3\left(\tau_{z y}^{2}+\tau_{z x}^{2}+\tau_{y z}^{2}\right)}{\left\{1+\zeta^{2}-\zeta\right\}}\right]^{\frac{1}{2}} y d y+ \\
& \int_{y_{e p}}^{\frac{t}{2}}\left[\frac{\left(c \frac{2}{\sqrt{3}} \dot{m}\right)^{2}}{\left\{1+\zeta^{2}-\zeta\right\}}\left(\left[\left(\frac{y}{R}\right)^{2}+\gamma_{x y}^{2}+\gamma_{y z}^{2}\right]^{\frac{1}{2}}\right)^{2 n}-\right. \\
& 3 \tau z y 2+\tau z x 2+\tau y z 21+\zeta 2-\zeta 12 y d y
\end{aligned}
$$

$$
\begin{gathered}
\int_{0}^{y e p}\left[\frac{\left(E \frac{2}{\sqrt{3}}\right)^{2}}{\left\{1+\zeta^{2}-\zeta\right\}}\left[\left(\frac{y}{R}\right)^{2}+\gamma_{x y}^{2}+\gamma_{y z}^{2}\right]-\frac{3\left(\tau_{z y}^{2}+\tau_{z x}^{2}+\tau_{y z}^{2}\right)}{\left\{1+\zeta^{2}-\zeta\right\}}\right]^{\frac{1}{2}} y d y+ \\
P=\frac{\int_{y_{e p}}^{\frac{t}{2}}\left[\frac{\left(c \frac{2}{\sqrt{\xi}} \dot{m}\right)^{2}}{\left\{1+\zeta^{2}-\zeta\right\}}\left(\left[\left(\frac{y}{R}\right)^{2}+\gamma_{x y}^{2}+\gamma_{y z}^{2}\right]^{\frac{1}{2}}\right)^{2 n}-\frac{3\left(\tau_{z y}^{2}+\tau_{z x}^{2}+\tau_{y z}^{2}\right)}{\left\{1+\zeta^{2}-\zeta\right\}}\right]^{\frac{1}{2}} y d y}{\left[\left\{\left(1-\frac{x}{a}\right)\left(\frac{x-\tan \theta\left(r_{1}-U\right)}{1+\mu \tan \theta}\right)\right\}-\left\{\frac{\mu x}{\cos \theta}\left(\sin \theta-\frac{x^{\prime} \cos \theta}{U^{\prime}}\right)\right\}\right]}
\end{gathered}
$$

To get the value of P i.e. top roller force, from the above equation 21 along with the material properties and geometrical parameters of the machine setting, the value of coefficient of friction $\mu$ at roller plate interface is required. Value of angle $\theta$ can be calculated from the geometrical configurations of the machine setting. Integration involved 
in the numerator of the equation is to be evaluated by numerical integration as the complete solution cannot be obtained for the same. Equation 21 is used for studying the effects of various parameters involved in the conical bending process and reported in the subsequent section.

\section{Effect of Plate Thickness and Coefficient of Friction on Dynamic Bending Force}

To study the effect of thickness of the plate on the dynamic bending force, analytical values of bending force have been evaluated for constant values of bottom roller inclination by varying the coefficient of friction and plotted as shown in figure 4 (a-e). Dynamic bending force for different thickness of the plates is also plotted on the same graph as shown in figure 4 (a-e).

It can be observed from the figure 4 that as the thickness of the plate increases for constant value of bottom roller inclination and constant value of coefficient of friction, the dynamic bending force increases. It is quite obvious as the plate thickness increases the material required to be bent will increase, which in turn increase the dynamic bending force.

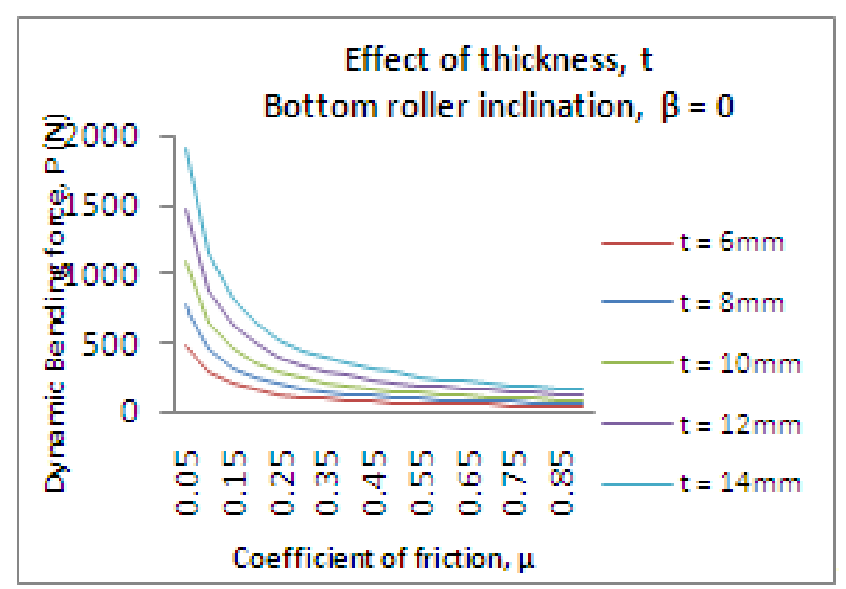

(a)

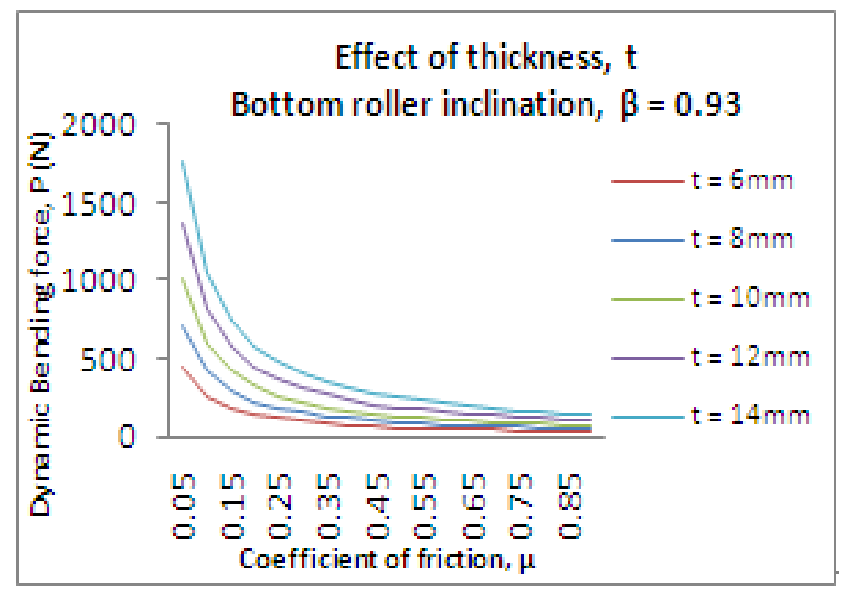

(b)
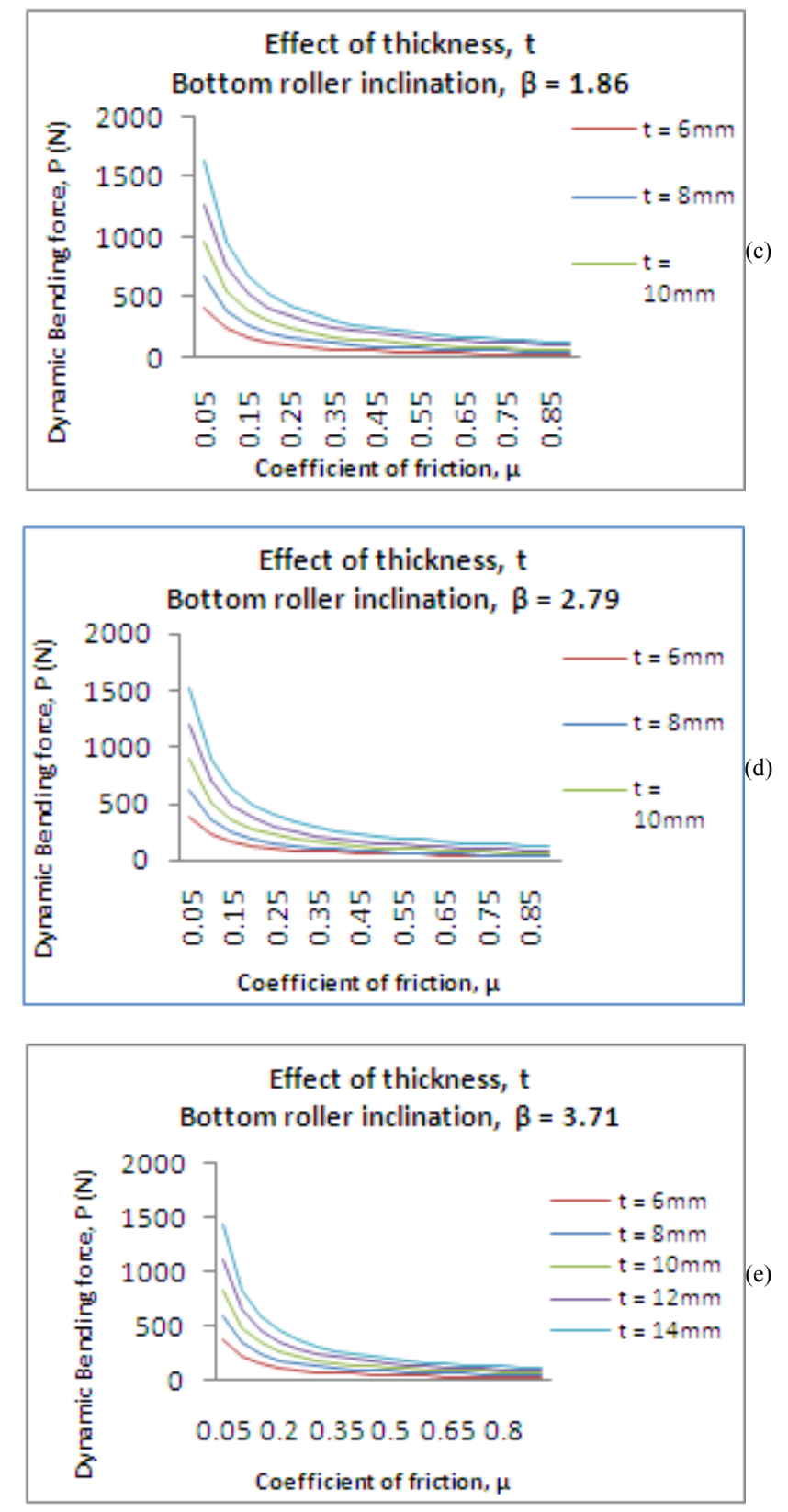

Figure 4. Effect of coefficient of friction and thickness on dynamic bending force

It can also be observed from figure 4 that the dynamic bending force reduces when the coefficient of friction increases for constant bottom roller inclination and constant thickness of the plate. If it is compared with the effect of coefficient of friction on static bending force [12], it is quite opposite to it. The bending force, increases in case of static bending when the coefficient of friction increases whereas the dynamic bending force decreases, as the coefficient of friction increases.

In figure 2 the bottom rollers are rotated in anti-clockwise direction and the frictional force are favoring the rotation of the roller. The frictional force assists the movement of the plate over the rollers by pulling the plate along with it. If the friction coefficient is higher, it will give better gripping of 
the plate on the bottom rollers and hence it will reduce the requirement of the bending force. That is why when the coefficient of friction increases in case of dynamic bending, the bending force reduces.

It can also be observed from figure 4 that as coefficient of friction $\mu$ decreases, the force required to bend the plate increases sharply. This phenomenon is because of slipping of plate over the roller when $\mu$ is very less, nearly zero. The coefficient of friction $\mu$ being very less near zero, there will not be proper gripping of the plate over the rollers. To allow rolling of plate over roller with less friction, more bending load for the top roller is required, resulting in sharp increase in force at $\mu$ near zero.

\section{Effect of Strain Hardening Exponent on Dynamic Bending Force}

To study the effects of strain hardening exponent ' $n$ ' on dynamic bending force, the analytical values of dynamic bending force have been calculated by varying ' $n$ ' for constant bottom roller inclination keeping all other parameters constant. The plot of dynamic bending force with respect to strain hardening exponent for different thicknesses have been plotted as shown in figure 5 (a-e).

It can be observed from figure 5 that as the strain hardening exponent increases the bending force reduces for constant bottom roller inclination and constant plate thickness. It is similar to the effect of strain hardening exponent on the static bending force [12]. As the strain hardening exponent increases, the internal stress required to deforming the material decreases and hence the force required to deform the material decreases.

The slope of the bending force curves with respect to strain hardening exponent decrease as the thickness of the plate decreases and the bending force curves almost become linear for thickness $6 \mathrm{~mm}$ as can be seen figure 4 . It can be seen from figure 2 that inelastic region stress will vary linearly while in plastic region the stress will vary non-linearly. For higher thickness the plastic region will be larger as compared to elastic region and hence the force variation for higher thickness will be non-linear as compared to the variation of bending force for lower thickness.

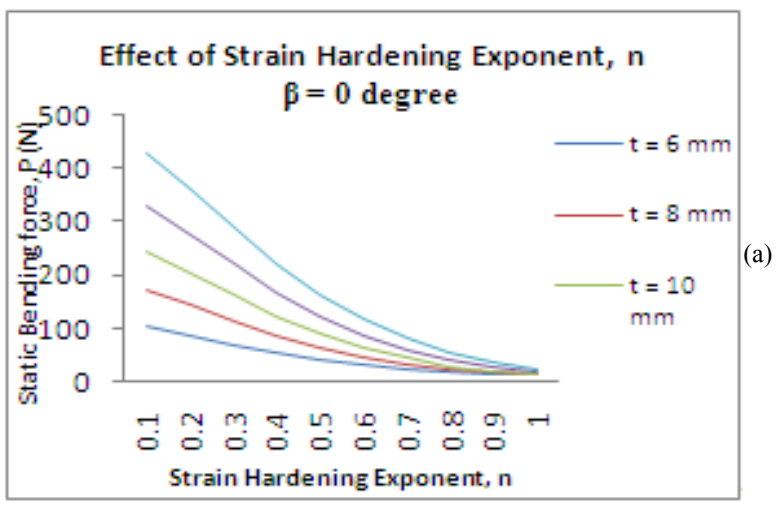

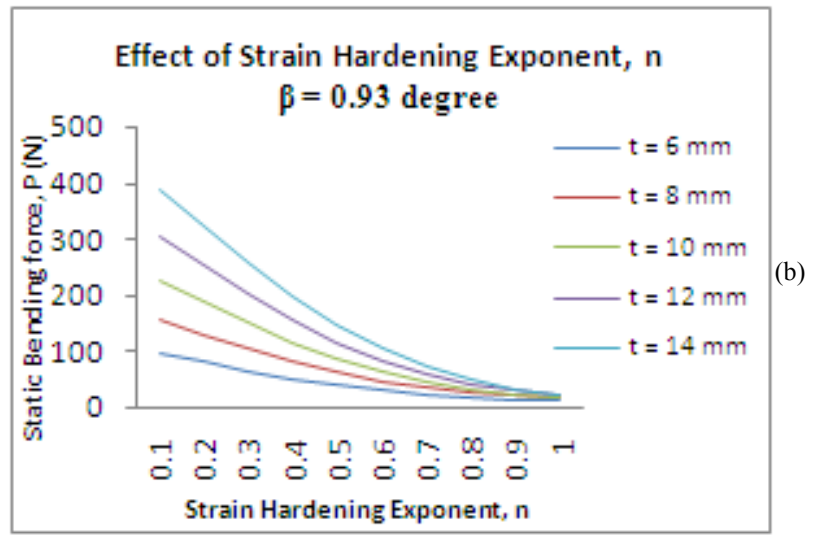
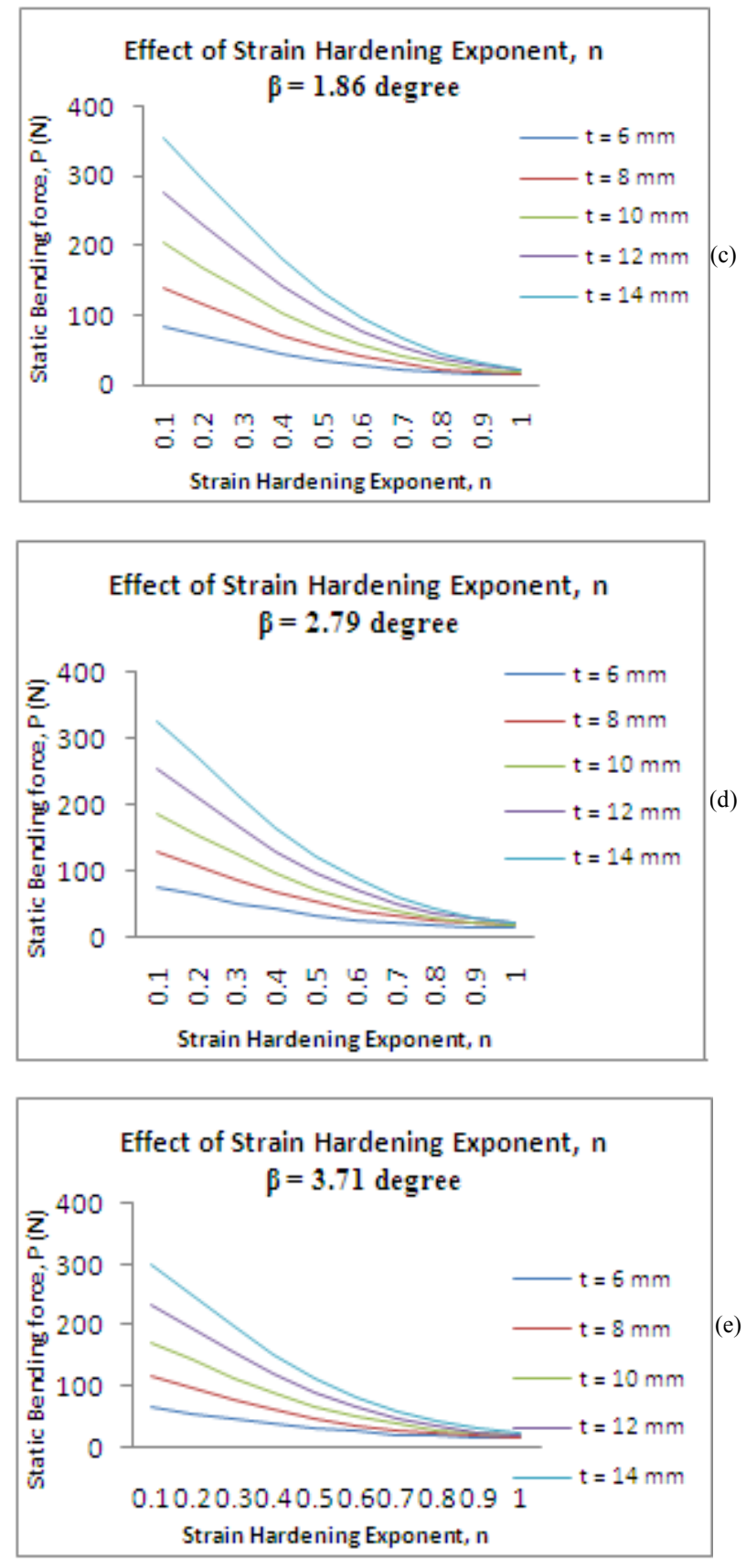

Figure 5. Effect of strain hardening exponent on the dynamic bending force 


\section{Conclusion}

Analytical model of bending force prediction for dynamic bending stage during 3-roller conical bending has been developed equating the external bending moment and internal bending moment. Shear stresses developed in the plate material were considered for the present derivation and effects of some material parameters have been studied. Following conclusions can be derived from the present study:

1. The analytical model of force prediction during dynamic roll bending has to be solved by the numerical methods and hence it will not give the exact solution. However, the model can be used to study the effects of various parameters on dynamic bending force.

2. As the thickness of the plate increases, the bending force required during dynamic bending increases and it represents the practical situation. In practice, it is observed that as the thickness increase the force required to bend the plate increases.

3. The friction at the roller plate interface improves grip of the bottom roller over the plate. The frictional forces assist the rollers in bending plate in dynamic conditions and hence as the coefficient of friction increases the dynamic bending force decreases.

4. Dynamic bending force decreases as the strain hardening exponent increases. This effect of strain hardening exponent is similar for static bending force.

5. Work carried out in the present paper will provide better understanding of the complex mechanics involved during the 3-roller conical bending process to the researchers working in the area of metal forming.

\section{Acknowledgement}

This research work is carried out with the financial support of Department of Science and Technology, Government of India, under SERC scheme.

\section{REFERENCES}

[1] M. Hua, Y.H. Lin, (1999), Large deflection analysis of elastoplastic plate in steady continuous four-roll bending process, International Journal of Mechanical Sciences, Vol. 41, Pages 1461-1483

[2] A. H. Gandhi, (2009) Investigation on machine setting parameters for 3-roller conical bending machine for springback, Ph. D. Thesis, S V National Institute of National Institute of Technology

[3] M. B. Basset and W. Johnson, (1966) The bending of plate using a three-roll pyramid type plate bending machine, J. Strain Anal. Eng. Des. Vol 1 No. 5 Pages 398-414

[4] N. E. Hansen, O. Jannerup, (1979) Modelling of elastic-plastic bending of beams using a roller bending machine, J. Eng. Ind. Vol 1013 Pages 304-310

[5] E. A. Kulikov, Y. I. Berliner, G. I. Shevykov, (1969)Bending thick walled conical vessel sections on roll bending machines, Chem. Pet. Eng.Vol 5 No12 Pages 977-979

[6] Hyunok Kim, Ninad Nargundkar, Taylan Altan, (2007) Prediction of bend allowance and springback in air bending, Trans. Of ASME, J. Mfg. Sci. Eng., Vol 129 Pages 342-251

[7] M. Samuel, (2000) Experimental and numerical prediction of springback and side wall curl in U-bendings of anisotropic sheet metals, Journal of Materials Processing Technology Vol 105 Pages 382-393

[8] M. Hua, I.M. Cole, K. Baines, K.P. Rao, (1997) A formulation for determining the single-pass mechanics of the continuous four-roll thin plate bending process, Journal of Materials Processing Technology,Vol 67 Pages 189-194

[9] M. Hua, D.H. Sansome, K. Baines, (1995) Mathematical modeling of the internal bending moment at the top roll contact in multi-pass four-roll thin-plate bending, Journal of Materials Processing Technology Vol 52 Pages 425-459

[10] Raval H. K., (2002) Experimental and theoretical investigation of bending process, (Vee and three roller bending), Ph.D. thesis, South Gujarat University, India

[11] A. H. Gandhi, H. K. Raval, (2008) Analytical and empirical modeling of top roller position for three-roller cylindrical bending of plates and its experimental verification, J. Mater. Process. Techno.Vol 197 Pages 268-278

[12] M. K. Chudasama, H. K. Raval, (2013) An approximate bending force prediction for 3-roller conical bending process, International Journal of Material Forming, Vol 6 No 2 Pages 303-314

[13] G. Martin, S. Tsang, (1966) The plastic bending of beams considering die friction effects, J. Eng. Ind. Pages 234-250

[14] R. Hill, (1950) The Mathematical theory of Plasticity, Oxford University Press

[15] V. V. Hasek, (1977) An evaluation of the applicability of Theoretical Analyses to the Forming Limit Diagram, Proc. Of 4th Int. Conf. On Fracture Vol 2 Pages 476-485

[16] W. F. Hosford, R. M. Caddel, (1993) Metal forming mechanics and metallurgy, Prentice Hall, NJ, USA

[17] Z. Marciniac, J.L. Duncan, S. J. Hu, (1992) Mechanics of sheet metal forming, Butterworth-Heinemann 